\title{
PRIMARY FACTORIZATION IN A WEAK BEZOUT DOMAIN
}

\author{
R. A. BEAUREGARD AND R. E. JOHNSON
}

\begin{abstract}
It is well known that in a weak Bezout domain each prime factorization of an element is unique up to similarity. In this paper, a corresponding extension to primary factorizations is ob-
\end{abstract} tained.

An integral domain with unity is called a weak Bezout domain ${ }^{1}$ by P. M. Cohn in [2] iff the sum and intersection of any two principal right ideals that have nonzero intersection are again principal. There it is shown that in such a ring any prime factorization of an element is unique up to order of factors and similarity. This generalizes the familiar result for commutative weak Bezout domains (called Bezout rings) that any prime factorization of an element is unique up to order of factors and associates. Just as in the commutative case one also considers primary decompositions, and when they exist the question of uniqueness arises. In this note we study primary factorizations in a weak Bezout domain, and we show that any primary factorization of an element is unique up to order of factors and similarity (associates for the commutative case).

In what follows $R$ denotes a weak Bezout domain, $R^{*}=R \backslash\{0\}$, and $U$ denotes the group of units of $R$. We assume the reader is familiar with the gcld $(a, b)_{l}, \operatorname{lcrm}[a, b]_{r}$, etc., and similarity $a \sim b$ of $a$, $b \in R^{*}$. In particular, if $a b^{\prime}=b a^{\prime}$ in $R^{*}$ then $(a, b)_{l}=\left(a^{\prime}, b^{\prime}\right)_{r}=1$ iff $[a, b]_{r}=\left[a^{\prime}, b^{\prime}\right]_{l}=a b^{\prime}=b a^{\prime}$, in which case $a \sim a^{\prime}$ and $b \sim b^{\prime}$. We call $c$ an $S$-factor of $b$ iff $c \sim c^{\prime}$ and $c^{\prime}$ is a factor of $b$. Left and right $S$-factors are defined in the obvious way. If $p \in R^{*} \backslash U, p$ is called prime iff in every factorization $p=p_{1} p_{2}$ either $p_{1} \in U$ or $p_{2} \in U$. An element $b \in R^{*} \backslash U$ is called $p$-primary for some prime $p$ iff every prime factor of $b$ is similar to $p$ and every nonunit $r$ - or $l$-factor of $b$ has at least one prime factor. We call $c=c_{1} c_{2} \cdots c_{n}$ a primary factorization of $c$ iff each $c_{i}$ is $p_{i}$-primary for some prime $p_{i}$ and $p_{i} \nsim p_{j}$ whenever $i \neq j$. An element of $R^{*} \backslash U$ need not have a primary factorization even if it can be 1969.

Received by the editors December 12, 1968 and, in revised form, September 6,

AMS Subject Classifications. Primary 1615.

Key Words and Phrases. Weak Bezout domain, unique prime factorization, unique primary factorization.

1 The definition of weak Bezout domain is left-right symmetric [2], and can be weakened by omitting the requirement that the intersection be principal [4]. 
factored into primes. This is obviously the case in a power series ring in noncommuting indeterminates over a field.

The main result is the following theorem whose proof will follow from the lemmas below.

Theorem. If $R$ is a weak Bezout domain and $c \in R^{*} \backslash U$ has two primary factorizations $c=a_{1} a_{2} \cdots a_{m}=b_{1} b_{2} \cdots b_{n}$ then $m=n$ and $a_{i} \sim b_{i \sigma}$ for some permutation $\sigma$ of $\{1,2, \cdots, n\}$.

Lemma 1. If $a b^{\prime}=b a^{\prime},(a, b)_{l}=c$, and $\left(a^{\prime}, b^{\prime}\right)_{r}=c^{\prime}$ in $R^{*}$, then there exist $x, x^{\prime}, y, y^{\prime} \in R^{*}$ such that $a=c x, b=c y, a^{\prime}=x^{\prime} c^{\prime}, b^{\prime}=y^{\prime} c^{\prime}, x y^{\prime}=y x^{\prime}$, and $x \sim x^{\prime}, y \sim y^{\prime}$.

Proof. Obvious.

LEMMA 2. If $a b^{\prime}=b a^{\prime}$ and $(a, b)_{l}=1$, then every l-factor of $a$ is a $l-S$ factor of $a^{\prime}$. If $a b^{\prime}=b a^{\prime}$ and $a$ is prime, then $a$ is either a l-factor of $b$ or a l-S-factor of $a^{\prime}$.

Proof. If $a_{1} \in R^{*} \backslash U$ is a $l$-factor of $a$, then $\left(a_{1}, b\right)_{l}=1$ and $\left[a_{1}, b\right]_{r}$ $=a^{\prime} b^{\prime \prime}=b a_{1}^{\prime}, a_{1} \sim a_{1}^{\prime}$, for some $b^{\prime \prime}, a_{1}^{\prime} \in R^{x}$. Since $b a^{\prime} \in\left[a_{1}, b\right]_{r} R, b a^{\prime}$ $=b a^{\prime} c$ for some $c \in R^{*}$ and $a^{\prime}=a_{1}^{\prime} c$. The second part is now obvious.

Leмma 3. If $a b^{\prime}=b a^{\prime}$ and no nonunit $l$-factor of $b^{\prime}$ is a $r$-S-factor of $b$, then $b=a u$ for some unit $u$.

Proof. (See [1, Theorem 1] for a special case.) By Lemma 1, $y$ and $y^{\prime}$ must be units.

Lemma 4. If $p$ is a prime factor of $a b^{\prime}$ then $p$ is an S-factor of either $a$ or $b^{\prime}$.

Proof. Assume $a b^{\prime}=b p e$ and $a^{\prime}=p e$. In the notation of Lemma 1, $p e=x^{\prime} c^{\prime}$ and $p$ is either a $l$-factor of $x^{\prime}$ or a $l$-S-factor of $c^{\prime}$ by Lemma 2 . Hence, $p$ is either a $l$-S-factor of $x$ by Lemma 2 and thus an $S$-factor of $a$ or an $S$-factor of $b^{\prime}$.

LEMMA 5. If $c=b_{1} a_{1} d_{1}=b_{2} a_{2} d_{2}$ and no nonunit $l-(r-)$ factor of $a_{1}$ or $a_{2}$ is an $S$-factor of $b_{1}$ or $b_{2}\left(d_{1}\right.$ or $\left.d_{2}\right)$, then $a_{1} \sim a_{2}$.

Proof. We might as well assume $\left(b_{1}, b_{2}\right)_{l}=1$. If $m=\left[b_{1}, b_{2}\right]_{r}$, then $m=b_{1} b_{2}^{\prime}=b_{2} b_{1}^{\prime}, c=m c^{\prime}$, and $a_{1} d_{1}=b_{2}^{\prime} c^{\prime}, a_{2} d_{2}=b_{1}^{\prime} c^{\prime}$ for some $b_{i}^{\prime}, c^{\prime} \in R^{*}$ with $b_{i}^{\prime} \sim b_{i}$. By assumption, $\left(a_{1}, b_{2}^{\prime}\right)_{l}=\left(a_{2}, b_{1}^{\prime}\right)=1$. If $\left(d_{i}, c^{\prime}\right)_{r}=e_{i}$ with $d_{i}=d_{i}^{\prime} e_{i}$ and $c^{\prime}=c_{i}^{\prime} e_{i}$, then $a_{1} d_{1}^{\prime}=b_{2}^{\prime} c_{1}^{\prime}, a_{2} d_{2}^{\prime}=b_{1}^{\prime} c_{2}^{\prime}$ and $a_{i} \sim c_{i}^{\prime}$. Therefore, no nonunit $r$-factor of $c_{i}^{\prime}$ is a $l$-S -factor of $e_{i}$ and $c_{1}^{\prime}=c_{2}^{\prime} u$ for some unit $u$ by Lemma 3. Hence, $c_{1}^{\prime} \sim c_{2}^{\prime}$ and $a_{1} \sim a_{2}$.

Proof of The Theorem. If $a_{i}$ is $p_{i}$-primary and $b_{j}$ is $q_{j}$-primary, 
then for each $i$ there exists a unique $j$ such that $p_{i} \sim q_{j}$ by Lemma 4 . Thus, $m=n$ and $p_{i} \sim q_{i \sigma}$ for some permutation $\sigma$ of $\{1,2, \cdots, n\}$. In turn, $a_{i} \sim b_{i \sigma}$ by Lemma 5 .

Since similar elements are associates in commutative rings, we have the following.

CoROllaRy. Let $R$ be a commutative Bezout ring and let $c \in R^{*}$ have a primary decomposition. Then this is unique up to order of factors and associates.

We conclude with an example ${ }^{2}$ of a commutative Bezout ring with elements having a primary decomposition but no prime factorization.

For $i=1,2, \cdots, n$, let $p_{i}$ be the $i$ th prime integer and consider the subring $R_{i}$ of $Q[x]$ (polynomials in $x$ with rational coefficients) consisting of all polynomials $f$ such that $f(i)$ has a denominator prime to $p_{i}$. On changing the variable to $x-i$, we can verify as in [3] that $R_{i}$ is a Bezout ring. We claim that $R=\bigcap_{i=1}^{n} R_{i}$ is again a Bezout ring. For let $a^{\prime}, b^{\prime} \in R$, and let $d_{i}$ be the gcd of $a^{\prime}, b^{\prime}$ in $R_{i}$, then $d_{i}$ is determined up to unit factor in $R_{i}$, i.e. a rational number prime to $p_{i}$. Hence we can write $d_{i}=d^{\prime} p_{i}^{\alpha_{i}}$ for some $d^{\prime} \in Q[x]$, and it follows that $d=d^{\prime} p_{1}^{\alpha_{1}} \cdots p_{n}^{\alpha_{n}}$ is a gcd of $a^{\prime}, b^{\prime}$ in $R$. Thus $a^{\prime}=d a, b^{\prime}=d b$, and $a v_{i}$ $-b u_{i}=1\left(u_{i}, v_{i} \in R_{i}\right)$. Suppose we have found $u, v \in R_{1} \cap \cdots \cap R_{i-1}$ to satisfy

$$
a v-b u=1,
$$

then $f=\left(u-u_{i}\right) / a=\left(v-v_{i}\right) / b$ lies in $Q[x]$. Hence we can find a power product of $p_{1}, \cdots, p_{i-1}$, say $\gamma$, such that $\gamma f \in R_{1} \cap \cdots \cap R_{i-1}$ and a power of $p_{i}$, say $\delta$, such that $\delta f \in R_{i}$. Since $\gamma, \delta$ are coprime, there are integers $\lambda, \mu$ such that $\lambda \gamma-\mu \delta=1$. Then $f=\lambda \gamma f-\mu \delta f$ and writing

$$
\begin{aligned}
& u^{\prime}=u-\lambda \gamma f a=u_{i}-\mu \delta f a, \\
& v^{\prime}=v-\lambda \gamma f b=v_{i}-\mu \delta f b,
\end{aligned}
$$

we see that (1) holds with $u, v$ replaced by $u^{\prime}, v^{\prime}$, and by (2) the latter are in $R_{1} \cap \cdots \cap R_{i}$. By induction we find $u, v \in R$ to satisfy (1) and this shows $R$ to be a Bezout ring.

In $R, x-i$ is $p_{i}$-primary but not a product of primes, and e.g. $x(x-1) \cdots(x-n)$ has a primary factorization.

\section{BIBLIOGRAPHY}

1. R. A. Beauregard, Infinite primes and unique factorization in a principal right ideal domain, Trans. Amer. Math. Soc. 141 (1969), 245-254.

2 We wish to thank the referee for communicating this example to us. 
2. P. M. Cohn, Noncommutative unique factorization domains, Trans. Amer. Math. Soc. 109 (1963), 313-331. MR 27 \#5785.

3. - Bezout rings and their subrings, Proc. Cambridge Philos. Soc. 64 (1968), 251-264. MR 36 \#5117.

4. R. E. Williams, $A$ note on weak Bezout rings, Proc. Amer. Math. Soc. 19 (1968), 951-952. MR 37 \#1108.

University of Rhode Island, Kingston, Rhode IsLand 02881 and

University of New Hampshire, Durham, New Hampshire 03824 